\title{
OCLC, WorldCat und die Metadaten-Kontroverse
}

\section{Adrian Pohl}

Mit der Ankündigung einer "Policy for Use and Transfer of WorldCat Records" ${ }^{\text {11 }}$ hat OCLC (Online Computer Library Center) in der angelsächsischen Bibliothekswelt eine lebhafte Diskussion hervorgerufen. Im deutschsprachigen Raum hat die geplante Policy allerdings bisher sehr wenig Resonanz gefunden. Ein Grund mag darin liegen, dass OCLC in Europa (noch) deutlich weniger Gewicht hat als in den USA. Sicher hätte das Inkraftreten einer OCLC-Metadaten-Policy (ganz gleich, wie sie ausgestaltet sein mag) weitreichende Auswirkungen auf das weltweite Bibliothekswesen. Eine Beschäftigung mit dem Thema ist also mehr als angebracht. Dieser Artikel dient dem Zweck, den Stand der Diskussion im angelsächsischen Raum wiederzugeben und einen Einblick in die verschiedenen Aspekte der Auseinandersetzung zu geben.

\section{Hintergründe}

Das Online Computer Library Center (OCLC) mit Sitz in Dublin (Ohio) präsentiert sich als eine Non-Profit-Mitgliederorganisation ${ }^{2}$, "die sich im öffentlichen Interesse für den breiteren, computergestützten Zugang zum weltweiten Wissen und die Senkung der damit verbundenen Kosten einsetzt. ${ }^{13}$ Im globalen Maßstab ist

1 Im weiteren Text wird auch von der "OCLC-Policy" oder nur der "Policy" gesprochen. Ich beziehe mich mit diesen Ausdrücken auf die aktuelle Version des Entwurfs einer entsprechenden Metadaten-Policy. Zur Entstehung und mehrfachen Überarbeitung der Policy vgl. Abschnitt 3.

2 Der Non-Profit-Status von OCLC wird von Kritikern immer wieder in Frage gestellt, vgl. z.B. Beall (2008). Offensichtlich wurde OCLC 1984 sogar der Non-Profit-Status von einem Gericht aberkannt und infolge einer nachträglichen Gesetzesänderung wieder hergestellt, vgl. Spalding (2008). Leider ist ohne Weiteres keine anständige Zusammenfassung dieses Vorgangs verfügbar. Siehe auch http://community.oclc.org /metalogue/archives/2008/11/notes-on-oclcs-updated-record.html\#comment-824, wo Karen Calhoun dazu Stellung nimmt, dass die Non-Profit-Organisation OCLC ForProfit-Unternehmen aufgekauft hat. Da sich in diesem Text der größte Teil der Referenzen auf Internet-Quellen bezieht, ist es ratsam, den online veröffentlichten Preprint dieses Artikels unter www.uebertext.org bei der Hand - oder besser "auf dem Monitor" - zu haben, um den Links zügig folgen zu können. Für alle InternetQuellen gilt: letzter Zugriff am 12. Februar 2009.

3 http://www.oclc.org/de/de/about/default.htm. Weitere allgemeine Informationen zur Geschichte von OCLC und WorldCat finden sich in Dugall (2007). Eine polemische, nichtsdestotrotz lesenswerte Auseinandersetzung mit OCLC findet sich in Beall (2008). 
OCLC die größte Organisation im Bibliothekswesen, mit knapp 70. 000 Mitgliedsbibliotheken in über 100 Ländern. ${ }^{4}$

Das Fundament von OCLC bildet der WorldCat, dessen Konzept dem eines Verbundkatalogs entspricht: eine bibliografische Online-Datenbank für die gemeinsame Katalogisierung, in der auch die Bestandsdaten der beteiligten Bibliotheken erfasst sind. Auf dieser Datenbank baut eine große Zahl der OCLC-Dienstleistungen auf, seien dies Katalogisierungs- und Metadatendienste, Recherchewerkzeuge für Endnutzer, Fernleihdienste oder Bestandsanalyse- und -managementwerkzeuge. ${ }^{5}$ Für die Teilnahme am WorldCat sowie die Fremddatenübernahme aus dem WorldCat berechnet OCLC den Mitgliedsinstitutionen nicht unbeträchtliche Summen. Die Einnahmen aus WorldCat-Gebühren und Fremddatendiensten machen mehr als ein Drittel der Gesamteinnahmen OCLCs aus: Im Steuerjahr 2007/2008 betrugen die Einnahmen OCLCs aus Metadatendiensten nach Unternehmensangaben 85,8 Millionen US-\$. Das sind knapp 35\% der Gesamteinnahmen von 246,4 Millionen US-\$ im selben Jahr. ${ }^{6}$ Man kann OCLC also ein großes finanzielles Interesse daran unterstellen, diesen Datenpool weiterhin unter eigener Kontrolle zu halten, damit die gewohnten Gewinnströme nicht versiegen.

Vor diesem Hintergrund nimmt es nicht wunder, dass OCLC versucht eine Policy einzuführen, welche die Geldströme auch in Zukunft sichern soll. Mit der Ankündigung dieser rechtsverbindlichen Regelung hat OCLC Anfang November 2008 die Gemüter kritischer Bibliothekare und von Open-Data-Verfechtern erhitzt. Viele Passagen der Policy erwecken den Eindruck, dass sich OCLC ein Monopol auf die WorldCat-Daten sichern will und Konkurrenz auszuschalten versucht. Die Reaktionen - besonders in der US-amerikanischen Blogosphäre ${ }^{7}$ - waren harsch, wodurch bereits einige Änderungen der Policy erreicht worden sind. Mitte Januar hat OCLC nun als Erwiderung auf die vehemente Kritik den geplanten Termin des Inkrafttretens der Policy nach hinten verlegt: von Mitte Februar auf das Dritte Quartal 2009. Mit dem Review "Board of Shared Data Creation and Stewardship" hat OCLC zudem ein Gremium einberufen, das indessen mit OCLC-Mitgliedern und anderen Beteiligten in Kontakt treten soll, um den Policy-Entwurf zu überarbeiten. ${ }^{8}$

4 Vgl. OCLC (2008 d), S. 6 und S. 8.

5 Vgl. die vollständige Liste der OCLC-Dienste unter http://www.oclc.org/services/a-to-z.htm

6 Vgl. OCLC (2008 d), S. 7 und S. 50.

7 Eine exzellente Übersicht über die Reaktionen auf die Policy bietet die Code4Lib-WikiSeite http://wiki.code4lib.org/index.php/OCLC_Policy_Change.

8 Vgl. OCLC (2009). Eine Liste der Mitglieder des Review-Boards findet sich unter http://www.oclc.org/worldcat/catalog/policy/board/default.htm.

Zu einer detaillierten Chronik der Policy-Entstehung vgl. Abschnitt 3. 
Was steht in der Policy? Wie rechtfertigt OCLC ihren Inhalt? Wie genau verlief der umstrittene Entstehungsprozess? Welche Punkte werden kritisiert? Auf welcher rechtlichen Basis soll die Policy wirksam sein? Und welche Bedeutung hat die Kontroverse für den deutschsprachigen Raum? Zu all diesen Fragen versucht der vorliegende Text Informationen, Fakten und Quellen wenn nicht Antworten zu liefern.

\section{Der Inhalt der Policy}

Auf den OCLC-Webseiten wird der Inhalt des Policy-Entwurfs wie folgt zusammengefasst:

„Es ist erlaubt:

1. WorldCat-Daten zu nutzen, zu kopieren, in andere Werke aufzunehmen und wiederzugeben.

2. WorldCat-Daten des Bestands Ihrer eigenen Bibliothek, Ihres Archivs oder Museums zu übertragen.

Unter folgenden Bedingungen:

1. Nichtkommerzieller Gebrauch: Die Nutzung von WorldCat-Daten zu kommerziellen Zwecken erfordert ein separates Übereinkommen mit OCLC.

2. Nichtkommerzieller Transfer: WorldCat-Daten dürfen nicht verkauft, unterlizenziert oder auf andere Weise gegen Gebühr, zu Gewinnzwecken oder zu kommerziellen Zwecken transferiert werden.

3. Kennzeichnung: OCLC hält Sie an, WorldCat und OCLC Inc. als Quelle von WorldCat-Datensätzen zu kennzeichnen.

4. Angemessene Nutzung: Eine Nutzung von WorldCat-Daten darf nicht andere davon abschrecken, bibliografische Daten oder Bestandsdaten zum WorldCat beizusteuern. Außerdem darf mit einer Nutzung nicht substanziell die Funktion, der Zweck und/oder die Größe von WorldCat nachgebildet werden.

5. Modifikation: OCLC hält Sie an, die OCLC-Nummer, den Link zur Policy und alle anderen Mittel der Zuschreibung von Daten zum WorldCat, nicht zu löschen.

6. Übertragung: Die Policy-Bestimmungen und -bedingungen bleiben auch nach jedem Transfer von WorldCat-Daten für diese Daten gültig. ${ }^{9}$

Zu den ersten beiden Punkten: Kommerzieller Gebrauch erfordert also ein Übereinkommen mit OCLC ${ }^{10}$ und einen Transfer der Daten zu kommerziellen Zwecken darf nur OCLC selbst durchführen.

9 Übersetzung der englischen Zusammenfassung der Policy von A.P., vgl. http://www.oclc.org/worldcat/catalog/policy/.

10 Um das Zustandekommen von Vereinbarungen zum kommerziellen Gebrauch von WorldCat-Daten zu erleichtern, hat OCLC sich die WorldCat-Record-use-Form ausgedacht, siehe OCLC (2008 b), S. 5 ff. und https://www3.oclc.org/app/worldcat/recorduse/. 
Die Punkte 3 und 5 hatten sich von Bestimmungen in den ersten Versionen des Policy-Entwurfs zu Empfehlungen in späteren Versionen gewandelt. OCLC beabsichtigt, ein MARC-Feld (996) zu jedem WorldCat-Datensatz hinzuzufügen, in dem der Status der Daten als "WorldCat-derived" deklariert ist und auf die jeweilige Version der Policy verwiesen wird. Ursprünglich sollte dieses Feld eben nicht aus dem Datensatz entfernt werden dürfen, während dies nunmehr erlaubt sein soll und OCLC nur noch dazu ermutigen will die Angaben beizubehalten.

Interessant und kontrovers sind besonders die Punkte 4 und 6 . Schon die hier wiedergegebene Zusammenfassung zeigt die möglicherweise weitreichenden Implikationen einer solchen Policy auf. Das Verbot eines Transfers von Metadaten zu WorldCat-ähnlichen Diensten erweckt klar den Eindruck, dass sich OCLC mit diesen Vorgaben etwaige Konkurrenten vom Hals halten möchte. Es wird schlichtweg verboten, mit WorldCat-Daten einen Dienst zu füttern oder aufzubauen, der eine Ähnlichkeit mit dem WorldCat hat.

Punkt 6 spiegelt die Anleihen der Policy an eine Creative-Commons-Lizenz wider. Ähnlich einer solchen CC-Lizenz hat die OCLC-Policy einen viralen Charakter, d.h. die Lizenzbedingungen und -bestimmungen werden - einem Virus ähnlich - gemeinsam mit dem Transfer der Daten übertragen und gelten somit auch für jeden Empfänger der Daten. Auf diese und andere kritische Punkte der Policy wird in Abschnitt 5 näher eingegangen.

\section{Kleine Chronik der Policy-Entstehung}

Hier soll nun ein kurzer Überblick über die Entstehung der "Policy for Use and Transfer of WorldCat Records" gegeben werden: ${ }^{11}$

- Der Auftrag: Das OCLC-Kuratorium (Board of Trustees) bittet das OCLCManagement um eine Aktualisierung der seit 1987 geltenden Guidelines for the Use and Transfer of OCLC-Derived Records. ${ }^{12}$

- Die Arbeitsgruppe: Die OCLC-Führung beauftragt im Januar 2008 Karen Calhoun, zu diesem Zweck ein Team - die "Record Use Study Group“ - zusammenzustellen.

- Umweltevaluation: Die „Record Use Study Group“ verschafft sich einen Überblick über Geschäftsmodelle einiger OCLC-ähnlicher Unternehmen (Ama-

11 Die Darstellung der ersten, nicht-öffentlichen Phase basiert größtenteils auf den Aussagen Karen Calhouns (Vizepräsidentin von OCLC, WorldCat und Metadatendienste) in ihrem Blog Metalogue, vgl. Calhoun (2008c). In einem Kommentar geht sie auf die Entstehung der Policy ein: http://community.oclc.org/metalogue/archives/2008 /11/notes-on-oclcs-updated-record.html\#comment-750

12 Vgl. OCLC (1987). Eben diese Guidelines - rechtlich unverbindliche Richtlinien - sollen nach dem Willen von OCLC Inc. durch eine rechtskräftige Policy ersetzt werden. 
zon, AllMusic, Pro-Quest, Twitter, Wikipedia, Sherpa/RoMEAO) ${ }^{13}$ und befragt Experten und Kollegen in anderen Unternehmen. ${ }^{14}$

- OCLC-interne Kommunikation: Im Oktober 2008 „erwähnt" Jay Jordan (der Präsident von OCLC) die neue Policy bei einer Sitzung des OCLC-Mitgliederrats (Members Council). ${ }^{15}$ Daraufhin findet eine Diskussion unter den Mitgliedern des Members Councils statt, die per E-Mail weitergeht. Diese Diskussionsphase vor Veröffentlichung der neuen Policy, die den Mitgliedern Kommentare und Kritik erlauben soll, dauert höchstens zehn Tage. ${ }^{16}$

- Information sickert durch: Ende November versendet NYLINK (eine Kooperative von Bibliotheken und Informationseinrichtungen im Staat New York) eine Nachricht an seine Mitglieder, in der die OCLC-Policy angekündigt wird. ${ }^{7}$ Diese E-Mail findet weite Verbreitung, woraufhin zunächst vor allem ein - nun herausgenommener - Teil des Policy-Entwurfs für Aufruhr sorgt, der besagt, dass der "Zugang zu und Benutzung von OCLC Online-Systemen durch jegliche Institution nach dem 2. November als Akzeptieren der neuen Policy gewertet" wird. $^{18}$

- Veröffentlichung und Rückzug der ersten Fassung der Policy: Am Sonntagmorgen des 2. Novembers wurde die erste Version der Policy auf den OCLC-Seiten veröffentlicht, allerdings noch am selben Tag wieder von der Seite genommen. ${ }^{19}$

13 Vgl. Calhoun (2008 a).

14 Darauf hinzuweisen ist, dass die Arbeit der Gruppe hinter verschlossenen Türen stattfand und für OCLC-Mitglieder wie interessierte Außenstehende nicht nachvollziehbar war. Es gab nie eine Webseite oder einen Einblick in die Erkenntnisse der Gruppe für OCLC-Mitglieder.

15 Das Treffen fand vom 19. bis 21. Oktober 2008 in Dublin, Ohio - dem Sitz von OCLC statt. In der vorab veröffentlichten Agenda des Treffens [OCLC (2008d)] wird die Policy mit keinem Wort erwähnt.

16 Linda Bills (eine stellvertretende Delegierte im Members Council) stellt den Ablauf als noch intransparenter dar. Vgl. ihren Kommentar in Metalogue unter http://community.oclc.org/metalogue/archives/2008/11/notes-on-oclcs-updated-record.html\# comment-775 Demnach sollten die Bibliotheksvertreter im Member's Council zunächst gar keinen Einblick in die neue Policy bekommen und hatten - nach Protestäußerungen - schließlich die Möglichkeit, einen Blick in die Policy zu werfen und Kommentare abzugeben, in einem Zeitrahmen von zehn Minuten.

17 Der Inhalt dieser E-Mail ist unter http://article.gmane.org/gmane.education.libraries. autocat/16951 einsehbar.

18 Im Original heißt es: „, access and use of OCLC online systems by any institution after November 2nd will be taken as acceptance of the new policy. " Vgl. Calhoun (2008 b), die E-Mail von OCLC in Reaktion auf die NYLINK-Ankündigung.

19 Eine archivierte Fassung der ersten Version findet sich hier: http://marc.coffeecode.net/oclc_2008_11_02/ 
- Am späten 4. November wurde die zweite Version der Policy ${ }^{20}$ veröffentlicht und am 19.11.2008 die aktuelle Version ${ }^{21}$.

- Die Resonanz: Seit der Veröffentlichung der ersten Version finden in einigen angelsächsischen bibliothekarischen Weblogs und Mailinglisten teilweise sehr lebhafte Diskussion zum Thema statt ${ }^{22}$ und es werden sogar zwei Internet-Petitionen ins Leben gerufen. ${ }^{23}$ Im Januar 2009 greift sogar The Guardian das Thema auf, was zeigt, dass die Diskussion eine immer breitere Öffentlichkeit findet. ${ }^{24}$ Im deutschsprachigen Raum findet das Thema - bis auf einige wenige Mitteilungen und Verweise in englischsprachige Blogs - wenig Resonanz. ${ }^{25}$

- Einberufung eines Revisionsgremiums und Verschiebung des Inkrafttretens: Am 13.1.2009 veröffentlicht OCLC eine Mitteilung, dass der OCLC Mitgliederrat (Members Council) und das OCLC Kuratorium (Board of Trustees) gemeinsam ein "Review Board of Shared Data Creation and Stewardship" einberufen werden, welches die OCLC-Mitglieder repräsentieren und OCLC im Hinblick auf die Prinzipien und Best Practice der Metadaten-Weitergabe beraten soll. Das Inkrafttreten der Policy wird auf das dritte Quartal 2009 verlegt. ${ }^{26}$ Bis dahin soll das neu gegründete Gremium Bibliothekare und andere Meinungsvertreter konsultieren, Berichte, Briefe, Blog-Einträge u.ä. prüfen, um dem Mitgliederrat Änderungsvorschläge zu unterbreiten.

20 Die Unterschiede zwischen der ersten und zweiten Version sind hier visualisiert: http://www.librarything.com/wiki/index.php?title=OCLC_Policy_Changes\&diff= 11748 \&oldid $=11747$

21 Die aktuelle Version ist über die OCLC-Webseite zugänglich, vgl. OCLC (2008a).

22 Diese Reaktionen sind ausführlich im Code4lib-Wiki dokumentiert, siehe http://wiki.code4lib.org/index.php/OCLC_Policy_Change.

23 Siehe unter http://watchdog.net/c/stop-oclc und unter http://www.petitiononline.com /oclc/petition.html.

24 Vgl. Grossman (2009).

25 Auf der großen deutschsprachigen Mailingliste für die Bibliothekswelt (Inetbib) etwa gab es bis Ende Januar noch keinerlei Erwähnung geschweige denn Auseinandersetzung mit der Policy. In den Weblogs Netbib (http://log.netbib.de/) und Jakoblog (http://jakoblog.de/) finden sich zumindest Hinweise auf die neue Policy sowie Links auf weiterführende Blogeinträge.

26 Vgl. OCLC (2009). 


\section{OCLCs Argumente für die Policy}

In den FAQs zur Policy ${ }^{27}$ nennt OCLC folgende Gründe für die Revision der seit 1987 geltenden "Guidelines for the Use and Transfer of OCLC-Derived Records"28:

- Die Welt war 1987 eine deutlich andere als jetzt. Auf die sich wandelnde Informationslandschaft soll die Policy angemessen reagieren.

- Die Sprache der Guidelines ist veraltet und muss modernisiert werden.

- Die Guidelines seien unklar in Bezug auf die Benutzung und Weitergabe von WorldCat-Daten, welche die Policy bereinigen soll.

- Die Policy soll die Möglichkeiten einer Weitergabe von WorldCat-Daten an OCLC-Mitglieder wie Nicht-Mitglieder vergrößern.

- Zudem nennen die FAQs folgenden Grund für die Einführung der Policy: „Reinforce OCLC's support for WorldCat data sharing that encourages innovation and benefits libraries, museums and archives while protecting OCLC's members' investment in WorldCat. ${ }^{.29}$

Die ersten drei Punkte sind trivial oder hätten auch durch Änderungen an den geltenden Guidelines umgesetzt werden können. Die beiden offensichtlichen Hauptgründe für die Entwicklung der Policy werden im letzten Punkt nur angedeutet und sollen im Folgenden näher erläutert werden.

\subsection{Die Policy als Grundlage der Web-Scale-Strategie}

Mit dem Verweis auf Innovation, die den Mitgliedsinstitutionen zugute kommen soll, spielt OCLC auf die "Web Scale“-Strategie des Unternehmens an. Diese interessante, von OCLC derzeit unablässig propagierte Strategie scheint wichtige Impulse für eine Policy gegeben zu haben. OCLC versucht seit einigen Jahren die Sichtbarkeit der Mitgliedsbibliotheken über das Internet zu vergrößern und nennt dies „building Web Scale for libraries"30. Diese Entwicklung begann mit Open WorldCat, und wird nun mit WorldCat.org ${ }^{31}$ und dessen Einbindung in Partner-

27 OCLC (2008 b), hier S. 1.

28 Zum Folgenden vgl. OCLC (2008 b), S. 1. Zu den OCLC Guidelines vgl. OCLC (1987).

$29 \operatorname{OCLC}(2008$ b), S. 1.

30 Vgl. OCLC (2008 c).

31 Oftmals werden diese sprachlich zu unterscheidenden Dimensionen des WorldCat WorldCat, Open WorldCat und WorldCat.org - durcheinandergebracht. Zur Erläuterung: WorldCat ist die von den Mitgliedsbibliotheken geteilte Datenbank (mit MARC21Datensätzen und Bestandsangaben), mit Open WorldCat wurde die Indexierung eines Teils der WorldCat-Daten in Google und Yahoo! bezeichnet, um die Auffindbarkeit von WorldCat-Daten über allgemeine Internetsuchmaschinen zu ermöglichen. WorldCat.org schließlich ist OCLCs Reaktion darauf, dass eben nicht alle WorldCat-Daten von Google und Co. indexiert wurden. Deswegen ging OCLC 2006 einfach mit einer eigenen Webseite an den Start, über die sich browserbasiert im WorldCat recherchieren lässt. 
seiten (das sind viel genutzte Internetdienste wie Google, Facebook etc.) weitergeführt. Und für Verhandlungen mit möglichen Partnerseiten sei eben rechtliche Klarheit über die Bedingungen einer Nutzung und Weitergabe der WorldCatDaten eine notwendige Bedingung:

"To be successful negotiating and working with prospective partners, many of which are in the private sector, OCLC needs to move beyond the Guidelines to a policy that will be recognized by these organizations and others outside the library-archives-museum space as a legal document. Achieving clarity about the rights and conditions for using and transferring WorldCat data is a precondition to OCLC's sitting down to talk, on its members' behalf, with organizations that otherwise might have little or no interest in promoting the use and visibility of library, archival, and museum collections and services." ${ }^{132}$

\subsection{Eine Policy zur Sicherung von Gewinnrückflüssen}

OCLC wie auch eine Menge Bibliothekare, die die OCLC-Politik verteidigen ${ }^{33}$, betonen regelmäßig: Die jahrzehntelangen Investitionen OCLCs und seiner Mitglieder in den WorldCat würden es notwendig machen, diesen Gemeinschaftswert zu schützen, um einen entsprechenden Rückfluss von Gewinnen zu gewährleisten. In den FAQs zur Policy wird dies so formuliert:

"OCLC member libraries have spent nearly 40 years supporting and contributing to WorldCat and the OCLC cooperative. With over 100 million records and over one billion holdings, WorldCat is the backbone of the cooperative and the services used by the membership. It is not economically feasible to maintain and expand WorldCat, its surrounding services and the cooperative on a completely open model." ${ }^{34}$

Damit wird ein Mittel, welches für eine gemeinschaftliche, kooperative Sache entwickelt und verwendet wurde, nachträglich zu einer Investition, zu einem exklusiven, marktfähigen Gut umgedeutet, das monetären Gewinn abzuwerfen habe. ${ }^{35}$

$32 \operatorname{OCLC}(2008$ b), S. 2.

33 Vgl. etwa Randall (2009): "I do believe that OCLC management has an obligation to the member organizations to protect the database, which is the collective work of the member cataloging agencies AND of the OCLC staff (on behalf of the members), and which is the single most valuable asset (being the entire foundation of the services provided by OCLC)."

34 OCLC (2008 b), S. 3, Punkt 6.

35 Karen Calhoun verwickelt sich in einem Blog-Eintrag in eine Vielzahl von Widersprüchen, als sie den WorldCat mit den Commons, der Allmende, vergleicht: "OCLC's and the members' central asset is the WorldCat database that we share. It is our common investment, our 'commons'. I believe it is the right course to protect the commons. Thus, as Garrett Hardin has suggested in his writings about the "tragedy of 
Diese Auffassung, dass die jahrzehntelangen Investitionen von Bibliotheken, Bibliothekaren und OCLC-Mitarbeitern in den WorldCat sich jetzt und in Zukunft rechnen müssten, ist häufig zu vernehmen und wird noch häufiger kritisiert. Sie ist - neben der "Web Scale"-Strategie OCLCs - der Hauptgrund für die Forderung nach einer solchen Policy.

\section{Die Kritik an der Policy}

In diesem Abschnitt werden die umstrittenensten Klauseln der "Policy for Use and Transfer of WorldCat Records" genauer betrachtet und die Kritik an innen erläutert. ${ }^{36}$

\subsection{Kritik am Zustandekommen der Policy}

Wie aus der Darstellung des Entstehungsprozesses der Policy ersichtlich ist, gibt es genug Gründe, diesen Prozess zu kritisieren. Der Verdacht liegt nahe, dass OCLC ursprünglich vor hatte, die Policy ohne großes Aufsehen "durch die Hintertür" einzuführen. So wurde die Policy weder in Kommunikation mit seinen Mitgliedern verfasst noch war der Entstehungsprozess transparent. Erst ein vorzeitiges Durchsickern von Informationen sowie ein Aufruhr in der Blogosphäre führten zu einer öffentlichen Diskussion der Policy und schließlich zur nun stattfindenden Konsultation der Mitglieder und anderer von der Policy Betroffener.

\subsection{Die OCLC-Policy als rechtswirksames Dokument}

Ein grundlegender Unterschied im Wesen des Regelwerks für den Umgang und den Transfer mit WorldCat-Metadaten ist, dass die bestehenden unverbindlichen Richtlinien ("Guidelines") durch ein rechtlich bindendes Dokument ersetzt werden sollen, auf dessen Basis theoretisch Mitglieder rechtlich belangt werden können. Wie Tim Spalding es formuliert: "The Policy transforms WorldCat in many

the commons," it is appropriate to regulate the use of the commons." Vgl. Calhoun (2008c). Der Vergleich indes ist völlig verfehlt: Die Commons in Hardins Artikel [vgl. Hardin (1968)] sind wirkliches Allgemeingut und nicht - wie der WorldCat - im exklusiven Besitz einer Gruppe, es ist schlichtweg sprachlich falsch, von den "Commons einer Gruppe“ zu sprechen. Außerdem ist die Pointe von Hardins Artikel, dass er über die Nutzung natürlicher Ressourcen verhandelt, d.h. über knappe Güter, wozu digitale Daten eben nicht gehören: Das wiederholte Kopieren und Verbreiten eines Datensatzes schränkt die Nutzbarkeit dieses Gutes durch andere nicht ein, weshalb es aus dieser Sicht keinen Grund gibt, die Weitergabe von digitalen Daten zu regulieren.

36 Für eine umfangreiche Sammlung von Kritikpunkten an der Policy, die diesem Abschnitt als Hauptvorlage diente, vgl. Spalding (2009). 
respects, but most of all in how OCLC relates legally to its members from a cooperative to a sort of licensure." ${ }^{37}$

In diesem Zusammenhang sollte man sich nicht irritieren lassen, wenn OCLC-Sprecher nur das Beste für OCLCs Mitglieder und die Bibliothekswelt im Ganzen versprechen. Auch den FAQs, die sich für Bibliotheken gar nicht so schlimm anhören, sollte nicht zuviel Aufmerksamkeit geschenkt werden. Letztlich wäre es allein der Inhalt der Policy, der in Zukunft bestimmen würde, was mit OCLC-Datensätzen gemacht werden darf und was nicht, zumindest gemäß dem aktuellen Entwurf: "This Policy is the final, complete and exclusive statement of the agreement of the partiwith respect to the subject matter hereof. ${ }^{\prime \prime 38}$

\subsection{Rückwirkende Geltung}

Der Geltungsbereich des derzeitigen Policy-Entwurf erstreckt sich auf sämtliche WorldCat-Daten, auch wenn ihre Katalogisierung fast vierzig Jahre vorher stattgefunden hat. OCLC will sich also nicht damit begnügen, nur den Umgang und Transfer der neu hinzukommenden Katalogdaten zu regeln. Es ist mindestens fraglich, ob eine solche Lizenzierung von Daten rechtens ist, die unter ganz anderen Bedingungen entstanden sind.

\section{4 Änderungen jeglicher Art sind jederzeit möglich}

Der Text des Policy-Entwurfs setzt fest, dass OCLC jederzeit Änderungen vornehmen und eine bestehende Version der Policy durch eine neue ersetzen kann:

"OCLC may issue a modified version of this Policy or a substitute for this Policy at any time and the modified or substitute version will apply to any Use and Transfer of WorldCat Records after the date of issuance (or other effective date specified by OCLC). ${ }^{139}$

OCLC hat also theoretisch die Macht, die Policy in Zukunft uneingeschränkt zu ändern.

\subsection{Bei Verstoß: Verbot der Benutzung von WorldCat-Daten}

Der Text des Policy-Entwurfs sagt eindeutig: Im Falle einer Nichteinhaltung der Bestimmungen verliert die Partei, die den Bestimmungen zuwidergehandelt hat, automatisch das Recht, WorldCat-Daten zu nutzen und zu übertragen. Dies würde im Prinzip die Schließung des jeweiligen Katalogs und damit der gesamten Bibliothek bedeuten. Der konkrete Wortlaut der Policy:

37 Vgl. ebd.

$38 \operatorname{OCLC}(2008 \mathrm{a}), \S \mathrm{E}, 7$.

$39 \operatorname{OCLC}(2008 \mathrm{a}), \S \mathrm{E}, 7$. 
"The rights to Use and Transfer WorldCat Records afforded by this Policy shall automatically terminate upon any breach of the terms of this Policy. ${ }^{140}$

Jede Art von Verstoß gegen die OCLC-Policy kann zu einem Verbot führen, WorldCat-Daten zu verwenden, selbst der kleinste Bruch der Bestimmungen. Besonders pikant ist diese Passage in Kombination mit der im nächsten Abschnitt erläuterten Klausel.

\subsection{Allein OCLC bestimmt, wann ein Verstoß vorliegt}

Abschnitt E, Paragraph 6 des OCLC-Policy-Entwurfs liest sich wie folgt:

"OCLC has the sole discretion to determine whether any Use and/or Transfer of WorldCatRecords complies with this Policy.141

Demnach liegt es allein in OCLCs Ermessen, wann jemand mit der Policy übereinstimmt oder eben dagegen verstößt. Daraus folgt in Kombination mit dem vorhergehenden Abschnitt, dass somit allein OCLC entscheiden könnte, wann einer Bibliothek die Nutzung von WorldCat-Daten untersagt wird.

\section{7 "Unangemessener" Gebrauch untersagt}

Laut dem derzeitigen OCLC-Policy-Entwurf steht jeder Gebrauch von WorldCatDaten, der kein "reasonable Use“ ist, nicht im Einklang mit der Policy und kann deshalb die entsprechenden Sanktionen nach sich ziehen. Spezifiziert wird ein „reasonable Use“" bzw. das, was nicht darunter fällt, in $\S$ B, 13 der Policy:

"'Reasonable Use' does not include any Use of WorldCat Records that:

a. discourages the contribution of bibliographic and holdings data to WorldCat, thus damaging OCLC Members' investment in WorldCat, and/or

b. substantially replicates the function, purpose, and/or size of WorldCat.".42

Berechtigterweise sehen viele Kritiker in diesem Abschnitt Hinweise darauf, dass OCLC sich Konkurrenten vom Leib halten will und versucht ein Monopol auf bibliografische Daten aufzubauen bzw. zu bewahren. Diese Bestimmung könnte etwa Diensten wie LibraryThing ${ }^{43}$ oder OpenLibrary ${ }^{44}$ gefährlich werden.

$40 \operatorname{OCLC}(2008 \mathrm{a}), \S \mathrm{E}, 1$.

$41 \operatorname{OCLC}\left(2008^{\circ}\right), \S \mathrm{E}, 6$.

$42 \operatorname{OCLC}(2008 \mathrm{a}), \S \mathrm{B}, 13$.

43 www.librarything.com.

44 http://openlibrary.org. Nach den Aussagen des technischen Leiters der Open Library, Aaron Swartz, hat OCLC in der Vergangenheit auf verschiedene Arten und Weisen versucht dem Konkurrenten zu schaden, indem Druck auf die OpenLibrary, ihre Finanzierer oder potenzielle Mitgestalter ausgeübt und auf Kooperationsanfragen nicht eingegangen wurde, vgl. Swartz (2008 a) und Swartz (2008 b). 


\section{8 Zum viralen Charakter der Policy}

"The policy transfers with the records", so fasst Roy Tennant den viralen Charakter der Policy zusammen. ${ }^{45}$ Das heißt, wann und von wem auch immer jemand WorldCat-Daten erhält, unterliegen jede weitere Nutzung und jeder Transfer der Daten den Bestimmungen der Policy. Das gilt auch für Institutionen, die von OCLC nie etwas gehört haben, geschweige denn irgendeinen Vertrag mit OCLC unterschrieben haben. In der Policy wird dies folgendermaßen formuliert:

"Regardless of the source from which WorldCat Records are received, Use and Transfer of WorldCat Records is authorized solely by OCLC pursuant to this Policy." ${ }^{146}$

Diesen viralen Charakter hat OCLC von den Creative-Commons-Lizenzen ${ }^{47}$ entlehnt. Karen Calhoun bemerkt dazu:

"The [Record Use] Study Group was particularly influenced by the Creative Commons set of licenses. It is no accident that the structure of OCLC's updated policy mirrors that of the Creative Commons Attribution-NoncommercialShare Alike 3.0 Unported license. ${ }^{148}$

Hier ist wichtig zu betonen: Es ist unabdingbar, auf ein Werk das Urheberrecht zu besitzen, um eine Creative-Commons-Lizenz zu verwenden. Bis heute ist es allerdings fraglich, ob OCLC den Policy-Entwurf auf Basis von Urheberrechtsansprüchen auf WorldCat-Datensätze verfasst hat. Auf jeden Fall ist es eine ungeklärte Frage, ob und wodurch OCLC das Recht hat, die WorldCat-Daten mit einer solchen viralen Lizenz zu versehen.

\section{Urheberrecht oder Dienstleistungsvertrag?}

Der letzte Abschnitt hat es angedeutet: Eine bis dato so ungeklärte wie wichtige Frage ist, unter welchen rechtlichen Rahmenbedingungen die OCLC-MetadatenPolicy Wirksamkeit beanspruchen will, konkret: Handelt es sich um eine Lizenz, die auf einem Urheberrechtsanspruch OCLCs an den Metadaten beruht oder soll die Policy Teil eines Dienstleistungsvertrages zwischen OCLC und seinen Mitgliedern/Kunden sein? ${ }^{49}$

45 Zu Deutsch in etwa: „Die Policy wird mit den Datensätzen übertragen.“ Roy Tennant in Wallis (2008), 24 Min. 43 Sek.

$46 \operatorname{OCLC}(2008 \mathrm{a}), \S \mathrm{E}, 5$.

47 Vgl. http://de.creativecommons.org/index.php.

48 Calhoun (2008c).

49 Der Großteil der Informationen an dieser Stelle speist sich aus einer Diskussion zum Thema auf der Mailingliste liblicense, vgl.

http://www.library.yale.edu/ Ilicense/ListArchives/0901/msg00115.html und folgende sowie http://www.library.yale.edu/ Ilicense/ListArchives/0902/msg00000.html und folgende, v.a. aber Richards (2009 b). 


\subsection{Eine urheberrechtsbasierte Lizenz?}

OCLC wurde schon verschiedentlich um eine Stellungnahme zu der Frage gebeten ${ }^{50}$, ob die Policy auf Urheberrechtsansprüchen basiert, eine Antwort steht aber immer noch aus. Da es hier vor allem um US-amerikanisches Urheberrecht geht, Urheberrechtsfragen meist sehr komplex sind, der Autor dieses Artikels kein Jurist ist und es sich hier auch noch um eine rechtliche Grauzone handelt, sollen an dieser Stelle nur einige Fakten zusammengetragen werden.

- OCLC hat schon mehrmals das Urheberrecht auf WorldCat als digitale Sammlung registriert. ${ }^{51}$

- Ob jemand ein Copyright auf einzelne bibliografische Datensätze oder auf Teile eines Datensatzes haben kann, ist bisher ungeklärt und Gegenstand zahlreicher Diskussionen. ${ }^{52}$

- Nach Ansicht des Juristen Robert C. Richards entspricht der Wortlaut der Policy gängigen Copyright-Lizenzen. ${ }^{53}$

- Offensichtlich versuchte OCLC schon Anfang der 1980er Jahre, das Urheberrecht auf WorldCat-Datensätze zu deklarieren - und scheiterte. ${ }^{54}$

Ganz egal, ob OCLC ein Urheberrecht auf einzelne WorldCat-Datensätze oder auf die WorldCat-Datenbank im Allgemeinen beansprucht, der Bibliothekswelt steht eine spannende Diskussion über den Status der von ihr produzierten Metadaten bevor. $^{55}$

50 Zum Beispiel von Richard Wallis (vgl. http://community.oclc.org/metalogue/archives /2008/11/notes-on-oclcs-updated-record.html\#comment-844).

51 Diese Information findet sich in der Copyright-Datenbank der Library of Congress. Einfach unter http://cocatalog.loc.gov eine Titelsuche nach „OCLC online bibliographic database" aufgeben. Vgl. Richards (2009 b).

52 Vgl. etwa diese Diskussion zum Urheberrecht auf bibliografische Daten im USamerikanischen Raum auf liblicense:

http://www.library.yale.edu/ Ilicense/ListArchives/0901/msg00115.html und http://www.library.yale.edu/ /license/ListArchives/0902/msg00000.html sowie die jeweiligen Antworten. Schon in den 80er Jahren des letzten Jahrhunderts gab es offensichtlich „numerous debates ... about the legality of [OCLC Inc.] copyrighting member records." Siehe http://www.bookism.org/open/2007/04/02/open-data-what-would-kilgour-think/.

53 Vgl. Richards (2009a).

54 Vgl. http://www.opencontentalliance.org/2008/11/05 /new-oclc-records-policy-generates-debate/\#comment-16.

55 Ironischerweise wird also jener Berufsstand, der sich den kostenlosen Zugang zu Schriften für jede Bürgerin auf die Fahnen geschrieben hat, darüber diskutieren, ob die von ihr selbst produzierten Metadaten frei zugänglich sein sollen. 


\subsection{Die Policy als Teil eines Dienstleistungsvertrags}

Die andere Möglichkeit, wie OCLC die Geltung der Policy sichern könnte, wäre diese: Die Policy gilt nur in Verbindung mit jenen Verträgen, die OCLC mit seine Kunden aushandelt, bevor diese OCLCs Dienste nutzen können. Was würde das bedeuten?

- Ist die Policy an einen Dienstleistungsvertrag gekoppelt, so kann sie für sämtliche WorldCat-Daten Geltung beanspruchen, auch wenn OCLC selbst kein Copyright auf diese Daten hat. ${ }^{56}$

- Der Dienstleistungsvertrag, in dessen Rahmen die Policy Geltung beanspruchen könnte, wäre etwa ein jährlicher Vertrag wie ihn OCLC mit seinen Kunden abschließt.

- Vorstellbar wäre auch ein Click-Through-Vertrag, der bei jeder Nutzung von OCLC-Diensten "unterzeichnet" werden muss. Allerdings wirft diese Art von Vertrag wiederum einige Probleme auf, so dass diese Variante sehr unwahrscheinlich ist. ${ }^{57}$

- Der virale Charakter der Policy wäre unter diesen Bedingungen sicher nicht durchsetzbar, weil dafür eben ein Urheberrecht notwendige Bedingung ist.

Zunächst ist es wichtig herauszufinden, auf welcher rechtlichen Basis OCLC die Policy durchsetzen möchte. Eine Diskussion über ein Urheberrecht auf bibliografische Daten scheint in diesem Zusammenhang unvermeidlich. Bibliotheken und Bibliotheksverbünde müssen sich klar darüber werden, wie sie mit ihren Daten umgehen wollen in Zeiten, wo es etliche Internetdienste gibt, die etwas mit den Daten anfangen können und wollen..$^{58}$

\section{Ausblick}

Die im US-amerikanischen Raum entstandene Diskussion über die OCLC-Policy ist zu begrüßen. Zudem zeigt der Streit um die Policy, dass OCLC solche wegweisenden Entscheidungen nicht ungestraft ohne Abstimmung mit seinen Mitgliedern durchsetzen kann. Es bestehen reelle Chancen, dass im Zuge der Diskussion die Mitgliedsbibliotheken erstarken und OCLC wieder zu einer wirklichen Mitgliederorganisation wird, so dass die Mitglieder über die Politik von OCLC Inc. entscheiden und ein Auge darauf haben, dass ihre Interessen und nicht die Interessen

56 Bei vielen WorldCat-Daten ist es sehr unwahrscheinlich, dass OCLC einen Urheberrechtsanspruch hat.

57 Vgl. dazu http://www.library.yale.edu/ Ilicense/ListArchives/0902/msg00044.html.

58 Dienste wie LibraryThing, Open Library oder auch \pm biblios (http://biblios.net) freuen sich über die kostenlose Bereitstellung von bibliografischen Daten. Der Gemeinsame Bibliotheksverbund (GBV) etwa bietet seine Daten schon LibraryThing-Nutzern zur Übernahme an - und nimmt auch am WorldCat teil... Vgl.

http://www.librarything.com/blog/2006/10/gbv-major-german-consortium-added.php. 
eines multinationalen Unternehmens gewahrt sind. Auch wenn die Aussagen von OCLC-Inc.-Repräsentanten dies immer wieder andeuten: Nicht alles, was gut für OCLC Inc. ist, ist auch zum Vorteil seiner Mitglieder.

Zum Ende des Artikels werden noch einige Implikationen dieser Debatte für den deutschsprachigen Raum angedeutet und relevante Fragen aufgeworfen. Wie die Diskussion ihren Fortgang nimmt, wird sich zeigen.

- Wie positionieren? Einige deutsche Bibliotheken (z.B. die Deutsche Nationalbibliothek) und Verbünde (HeBIS, GBV und SWB) nehmen am WorldCat teil, andere befinden sich in Verhandlungen mit OCLC (BVB, KOBV und hbz). Wie werden sie auf die Pläne OCLCs reagieren? Welche Position beziehen sie? Und wie wollen sie auf die Entwicklung einer Policy, deren Ausgestaltung oder auch Vereitelung Einfluss nehmen?

- Mögliche Konflikte: Der GBV und der SWB sind WorldCat-Teilnehmer und bieten ihre Daten auch LibraryThing-Nutzern via Z39.50 zur Übernahme an. Bei einem Inkrafttreten einer Policy wie sie der aktuelle Entwurf vorsieht wäre eine solche Praxis unter Umständen illegal, bzw. würde separate Abkommen mit OCLC erfordern.

- Alternativen entwickeln? Die Debatte um die Policy verunsichert sowohl OCLCMitglieder als auch solche Institutionen, die über eine Mitgliedschaft bei OCLC nachdenken. Es stellen sich Fragen wie: Gibt es Alternativen zum WorldCat und vor allem zu WorldCat.org? ${ }^{59}$ Sollten solche Alternativen entwickelt werden? Wenn ja, wer tut dies?

- Die Metadaten-Politik deutscher Bibliotheken: Wie stehen deutsche Bibliotheken im Allgemeinen zu einem Urheberrecht auf bibliografische Metadaten und zu einer Einschränkung des Zugangs auf diese Daten? ${ }^{60}$ Wie zum freien Zugang und zur kostenlosen Verbreitung von Metadaten? ${ }^{61}$

59 Interessant ist in diesem Zusammenhang sicher \pm biblios.net, eine Social-CatalogingPlattform und freie Datenbank bibliografischer Metadaten, die für kooperatives Katalogisieren entwickelt wurde und Anfang Januar dieses Jahres gestartet ist, vgl. http://biblios.net.

60 Gerade vor dem Hintergrund, dass in der deutschen Bibliothekslandschaft der Open Access zu wissenschaftlichen Texten und Forschungsdaten einige Förderung erfährt, wäre es widersinnig, wenn nicht mit den eigenen Daten genauso verfahren würde und stattdessen der Zugang zu bibliografischen Metadaten beschränkt würde.

61 Übrigens hat die Forderung nach freiem Zugang zu und der Verbreitung von bibliografischen Metadaten in der Bibliothekswelt eine lange Tradition. Schon der römische Bibliothekar Melvilius Devius forderte im 4. Jahrhundert: „Liberate Metadata Librorum!“ („Befreit die bibliografischen Metadaten!“), vgl. Devius:

Opera 3 1/3: De Libris Nostris. 


\section{Quellen}

Für alle Internetquellen gilt: letzter Zugriff am 12. Februar 2009.

Beall, Jeffrey (2008): OCLC: A Review in: Roberto, K. R.: Radical Cataloging: Essays at the Front. McFarland, pp. 85-93. (Online-Version verfügbar unter http://eprints. rclis.org/13701).

Calhoun, Karen (2008 a): Some Rights Reserved: The Environment for Data Sharing. Einsehbar unter http://www.slideshare.net/amarintha /calhoun-data-sharing-panel-ifla-aug-2008-presentation.

Calhoun, Karen (2008 b): OCLC's Policy for WorldCat Records. E-Mail vom 28.10.2008 an verschiedene Mailinglisten, u.a. AUTOCAT. Einsehbar unter http://permalink.gmane.org/gmane.education.libraries.autocat /16964.

Calhoun, Karen (2008 c): Notes on OCLC's Updated Record Use Policy. Blog-Beitrag einsehbar unter http://community.oclc.org/metalogue/archives/2008 /11/notes-on-oclcs-updated-record.html.

Grossman, Wendy M. (2009): Why you can't find a library book in your search engine in: The Guardian, 22. Januar 2009, Technology news \& features, S. 3. Einsehbar unter http://www.guardian.co.uk/technology/2009/jan/22 /library-search-engines-books.

Hardin, Garrett (1968). Tragedy of the Commons. In: Science, 162, S. 1243-1248. Eine pdf-Version des Artikels findet sich unter http://www.sciencemag.org/cgi /reprint/162/3859/1243.pdf.

OCLC (1987): Guidelines for the Use and Transfer of OCLC-Derived Records. Einsehbar unter http://www.oclc.org/support/documentation/worldcat/records /guidelines/default.htm.

OCLC (2008 a): Policy for Use and Transfer of WorldCat Records.

Einsehbar unter http://www.oclc.org/worldcat/catalog/policy/recordusepolicy.pdf.

OCLC (2008 b): Policy for Use and Transfer of WorldCat Records. Frequently Asked Questions.

Einsehbar unter http://purl.org/oclc/wcrup-faq.

OCLC (2008 c): „Web scale" discovery and delivery of library resources. Einsehbar unter http://www.oclc.org/de/de/worldcat/web/default.htm.

OCLC (2008 d): Agenda des OCLC Members Council Meeting October 19-21, 2008. Einsehbar unter http://www.oclc.org/memberscouncil/meetings/2009/october /200810agenda.pdf.

OCLC (2008 d): OCLC Annual Report 2007/2008.

Einsehbar unter http://www.oclc.org/de/de/enews/2008/07/de_annual.htm. 
OCLC (2009): OCLC Board of Trustees and Members Council to convene Review Board of Shared Data Creation and Stewardship. Nachricht vom 13. Januar 2009, einsehbar unter http://www.oclc.org/news/releases/20092.htm.

Randall, Kevin M. (2009): Re: [ACAT] SUPER-OPAC ON THE WEB Re: [ACAT] OCLC and the Guardian. Mailinglisten-Beitrag vom 28. Januar 2009 auf AUTOCAT Einsehbar unter http://article.gmane.org/gmane.education.libraries.autocat/19290.

Richards, Robert C. (2009 a): Re: Copyright in Bib Records.

Mailinglisten-Beitrag vom 3. Februar 2009, einsehbar unter http://article.gmane.org /gmane.education.libraries.autocat/19449.

Richards, Robert C. (2009 b): RE: OCLC's New License for Bibliographic Records. Mailinglisten-Beitrag vom 6. Februar 2009 auf liblicense, einsehbar unter http://www.library.yale.edu/ Ilicense/ListArchives/0902/msg00044.html.

Swartz, Aaron (2008 a): Stealing Your Library: The OCLC Powergrab. Blog-Beitrag vom 13.11.2008 einsehbar unter http://www.aaronsw.com/weblog/oclcscam.

Swartz, Aaron (2008 b): OCLC on the Run.

Blog-Beitrag vom 15.11.2008 einsehbar unter http://www.aaronsw.com/weblog /oclcreply.

Spalding, Tim (2009): Why libraries must reject the OCLC Policy (part 1). Blog-Beitrag vom 11.1.2009 einsehbar unter http://www.librarything.com /thingology/2009/01/why-libraries-must-reject-oclc-policy.php.

Wallis, Richard (2008): OCLC Talk with Talis about the new Record Use Policy. Podcast-Gespräch von Richard Wallis mit Karen Calhoun und Roy Tennant (beide OCLC), siehe http://blogs.talis.com/panlibus/archives/2008/11 /oclc-talk-with-talis-about-the-new-record-use-policy.php. 